\title{
Sandwich Structure Based On Corrugated-Core: A Review
}

\author{
N. Z. M. Zaid" ${ }^{1, a}$, M.R.M. Rejab ${ }^{2}$ and N.A.N Mohamed ${ }^{1}$ \\ ${ }^{1}$ Faculty of Mechanical Engineering, Universiti Malaysia Pahang, \\ 26600 Pekan, Pahang, Malaysia
}

\begin{abstract}
Sandwich structures are commonly based on polymeric foam and honeycomb core material, for use in lightweight applications such as fuselage in aero plane, hull in marine construction and others. A review of sandwich structure based on corrugated-core is proposed and presented in this paper. Firstly, this paper aims to provide a means of comparing available sandwich structure in industries. Secondly, this paper aims to provide sandwich structure with corrugated-core for future research development efforts in field of sandwich construction. This paper starts with introduction of composite material such as sandwich structure, the advantages of sandwich structure was shown. After that these papers provide the structure of sandwich structure which includes the two faces and the cores. Furthermore, sandwich structure with different cores, which is honeycomb, foam core and corrugated core are discussed. At the end, the paper discussed more on corrugated-core for future research development.
\end{abstract}

\section{Introduction}

Advanced composites materials such as sandwich structure have remained progressively substituting traditional materials such as aluminum and steel in various industries fabricating all from small part to the key structural components of heavyweight vehicle such as airplane and marine ship[1].

The advantages of sandwich structures include great stiffness to weight ratios [2-5], improved fatigue life, steadiness under compressive forces, good thermal and acoustic isolation properties. These benefits determine the fact that the use of composites has improved ominously in a wide range of structural applications and first of all in aerospace manufacturing [6-9].

Katzman et al. [10] said that sandwich structures are usually based on honeycomb core and polymeric foam materials may keep air and humidity. Moisture retention is one of the problems in aircraft sandwich construction. This problem may lead to growth in the whole weight of the sandwich construction and degrading of the core properties. To overcome problems, an open channel core material such as two-dimensional prismatic core is vent able in order to avoid moisture accumulation. In addition, the suitability of the corrugated-core as replacement core design structures in the sandwich construction will be as well serves the concept of sustainable manufacturing.

\section{Sandwich Structure}

Generally, single layer sandwich structure consists of three main parts that are a core and two face sheets. With

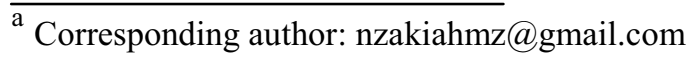

an extra sheet, called internal sheet, inserted into the core, a two-layer sandwich panel is then formed. Sandwich structure comprise of two relatively thin, stiff and strong faces divided by a relatively thick lightweight core, for example, honeycomb core, foam core and cellular metal. Stiff and simultaneous light component is very important in nowadays industries. To achieve lightweight and high stiffness component, sandwich structure construction is develop [1-4].

Depending on the determination of the materials can differ, nevertheless the most significant characteristics for sandwich constructions are, they are lightweight compared to metallic, high stiffness and cost effective compared to other composite structures. For specific applications using core material for example in aerospace, automotive, marine transportation, satellites, truck structures, containers, tanks, body parts, rail cars and wind energy systems, the construction technology used for the sandwich construction is significant $[\underline{1}, \underline{5}]$.

Sandwich structures are being considered for application to airplane main structures, durability and damage tolerance is a first rank contemplation, thus, understanding the opposing effect of in-service events. In fact, development of composite structure to sensitive fields, where high reliability is required, such as domestic flight, was so far restricted by the poor information of their behavior under intricate dynamic loads. Then, the structure needs to be assessed in order to verify that damage occurring during the service life will not lead to failure or extreme structural deformation until the damage is detected. The understanding of their static and fatigue behavior are essential in order to use the material in different application and a better considerate of the 
various failure mechanisms under static and fatigue loadings situations is essential and extremely necessary [5].

Because of their high specific strengths and stiffness, sandwich structures are extensively using in lightweight construction especially aerospace industries. Sandwich panel comprise of a lightweight core cover by two thin face sheets. Every face sheet may be an isotropic material or a fiber-reinforced composite laminate while the core material may either be of metallic/polymeric foam or metallic/aramid honeycomb [11]. The main profits of using the sandwich concept in structural components are the high stiffness and low weight ratio. These structures can carry out-of-plane and in-plane loads and show good steadiness under compression, possession excellent strength to weight and stiffness to weight characteristics. There are many benefits of sandwich constructions, the expansion of new materials and the essential for great performance and low-weight structures cover that sandwich structures will continue to be in demand $[\underline{1}, \underline{8}]$.

Polymers, wood, aluminum and composites are used for the core. These are to minimize weight that are used in form of foams, corrugated and honeycombs construction [12]. Sandwich structures with cellular core materials compromise great definite strength and an interest energy absorbing capacity. These sandwich structure properties make them a good answer for the protection of aircraft structure from impacting unknown objects. For instance, such panels are frequently used ahead of airplane to avoid unintended bird strikes, which can effect major damages to equipment and thus affect their safety. This shield is to avoid damage of panels which can cause the depressurization of aircraft [13].

\subsection{Faces/Skin}

The faces adhesively stuck to the core to gain a load transfer among the components. This method the properties of each detached component are utilized to the structural advantages of the whole assembly leading to a very high stiffness-to-weight and high bending strengthto-weight ratios. Typically, the facing layer realized by high pressure laminates, aluminum plates, and glass fiber reinforced plastics. Basically, the skins are thin, stiff and very strong. Figure 1 shows the example of skin part in sandwich structures.

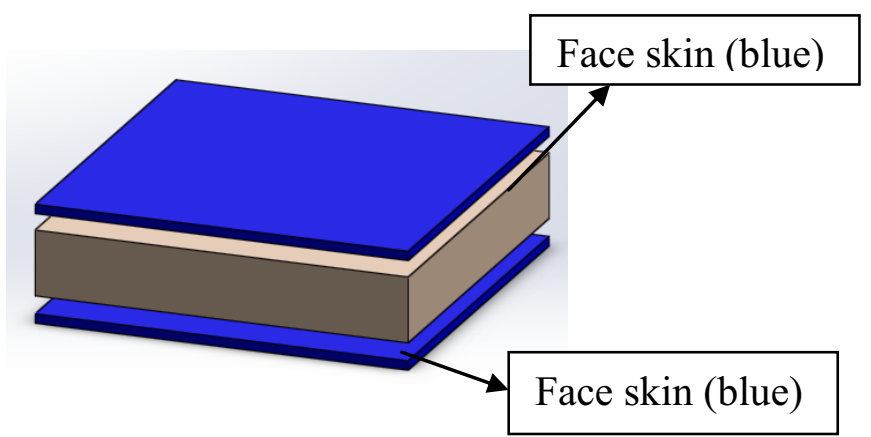

Figure 1: The skin of sandwich structure (Konka et. al 2012) [6]

\subsection{Core}

The sandwich structure core is the structure that placed between two thin faces. The material of the core is usually low strength material but its higher thickness provides the sandwich composite with high bending stiffness with overall low density. The purpose of the core is to backing the thin skin so that it does not deform and stay fixed relative to each other.

In recent years, there is several type of sandwich structures with better quasi-static and dynamic properties have been introduced, comprising those based on various foam, honeycomb cores, origami cores and truss cores [14].

\subsection{Honeycomb}

Aktay et al. [15] said that honeycomb is well-known core used to build sandwich structure. The name comes from the structure of honeycombs made by bees to store honey. Honeycombs and flex cores are used in many applications, for example chassis of modern cars use honeycomb sandwich structures. The honeycomb, flexcores and nomex are sandwiched between two carbon skins with the purpose of making a very stiff and strong structure that offers shield to the driver in case of a simple crash.

In aerospace applications, honeycomb materials have been broadly used among core materials. Various research have been conducted to know the mechanical response of honeycomb structures under dissimilar loadings [16].

Normally honeycomb has a uniform shape of hexagonal construction defined by the cell size, material, cell wall thickness and bulk density like Figure 2 below. The main materials for the core are glass fiber reinforced plastic, aluminum and aramid paper. Between them, aramid paper and aluminum are usually used in engineering application.

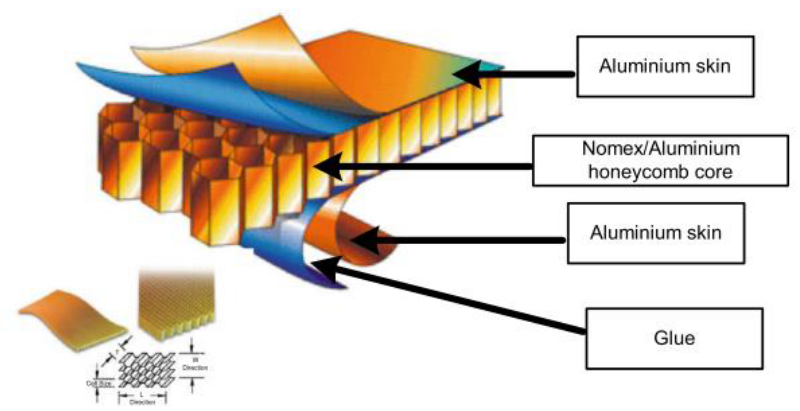

Figure 2: Example of Honeycomb sandwich structure (Abbadi et al. 2009)[17]

\subsection{Foam Core}

A big number of core materials and core configurations have been proposed these days. The most often used core materials are foams and honeycomb. The foam cores are preferably used when the waterproof, sound and heat insulation qualities of cores are required. Moreover, the foam cores are the cheapest among core materials and can offer some benefits in sandwich manufacture [18]. 
The stuffing of honeycomb cells with foam can be considered as the enhancement of debonding resistance and ability to produce new types of sandwich cores. This concept combines the benefits of honeycomb and foam cores. The increased adhesive area of foam-filled honeycomb cells is only one of them. On the other hand, the filling leads to changes of the dynamic properties of the honeycomb sandwiches [18].

Adding foam in the honeycomb core causes adequate decrease of the magnitudes of the natural frequencies. This effect is enlarged by the density of foam fillers, which trivially increases the stiffness and total mass of the filled sandwich plates. Filling with the foam promotes the slight increases of buckling loads and the insignificant decreases of imperfection sensitivity of foam-filled sandwich plates. Instead, adding the foam can make changes in stress distributions in the core to sheet faces interface [18].

The sandwich constructions have become more widely used thanks to the use of cellular foams as a structural element and load bearing component. Now day the main effort in design is put into face materials, but as the demands for more improved structures are raised, the research in the field of core materials is increased [19].

The situation of aluminum alloy foam with coldworked aluminum face sheets have been studied experimentally and theoretically for plastic collapse modes of sandwich beams. Plastic collapse is by three contending mechanisms: face yield, indentation and core shear, with the active mechanism provisional upon the choice of geometry and material properties [20].

Recently, Chen et al. [20] explained that a range of metal foams have been established with a relative density $\rho$ (defined by the ratio of foam density to the density of the cell walls) in the range 0.05 to 0.3 . Generally are based on aluminum alloys, though steel foams and creepresistant nickel alloy foams are also under progress. Metal foams compete favorably with polymer foams as the lightweight cores of sandwich beams, plates and shells, due to the higher stiffness and high-temperature ability.

Crupi et al. [21] discussed the aluminum foam sandwiches (AFS), gained by joining metal face sheets with a lightweight metal foam core, it have low specific weight, efficient capacity of energy dissipation, high impact strength, acoustic and thermal insulation, high damping, etc., that made them interesting for a number of practical applications, such as the realization of lightweight structures with high mechanical strength and good capacity of energy dissipation under impacts. When compared to traditional honeycomb panels, Aluminum Foam Sandwich offers several advantages. It can be prepared into curved shapes and with integral skins (without adhesive bonding), allowing higher working temperature and higher resistance to damage from water intrusion, which could be important for marine applications. Aluminum foams have potential to change polymer foams in sandwich panel applications due to their improved specific stiffness and higher temperature ability [2].

Usually, the low density cores of the sandwich structure are made of polymeric foams of numerous relative densities. Foam materials are however bending controlled structures, and the material is thus not fully developed, which results in a lower weight specific performance compared to a stretching controlled structure [23].

\subsection{Corrugated-core}

A corrugated-core sandwich structure is embraced of a corrugation sheet between two thin surface sheets. The important feature of this structure is its high strength-toweight ratio. The corrugated-core keeps the face sheets apart and stabilizes them by resisting vertical deformations, and also enables the whole structure to act as a single thick plate as an asset of its shearing strength. This second feature gives better strength to the sandwich structures. Furthermore, unlike soft honeycomb shaped cores, a corrugated-core opposes bending and twisting as well to vertical shear. Then, corrugated-core sandwich panels, due to their extremely high flexural stiffness-toweight ratio are usually used in aeronautics, aerospace, civil engineering and other applications, where weight is a significant design issue. This structure approach to form a sandwich plate may be defined as 'structurally composite', since its behavior characteristics are defined by the composite action of its components [24].

Rejab et al. [14] showed that corrugated cores when tested in the longitudinal direction offer shear strengths that are comparable with square honeycombs and significantly greater than those exhibited by diamond cores and more traditional foam cores. The second different feature of a corrugated-core is its ability to gives outstanding ventilation characteristics, avoiding problems related with humidity retention that is common in cellular core materials (e.g. polymeric foams and honeycombs). Humidity-retention can be a problem in many aerospace structures, e.g. aluminum honeycombs, but the adoption of corrugated, origami-type and truss core structures can minimize this problem.

Corrugated-cores with metal sandwich panels are an interesting industrial solution as structural components this is because their high stiffness-to-mass ratio. However, using detailed finite element models for numerical computation of their properties leads to large models and long solution time, specifically for acoustic simulations. Then, decrease of the complex shaped core to an equivalent homogenous material is usually used [25].

Amongst all sandwich panels, corrugated-core structures are an interesting alternative that is being progressively used in the transportation industry. For these panels there are dissimilar core shapes, such as truss-type corrugations (i.e. triangular), circular shape or trapezoidal cores [25].

Cote et al. [26] explain that prismatic, such as the $\mathrm{Y}$ core and NavTruss, are chosen in naval sandwich structure for two reasons (i) they are direct to construction on large length scales by a welding route and (ii) the high longitudinal elongating and shear strength of the cores makes them ideal for application in sandwich beams. 
Hou et al. [27] aims to investigate the effects of the key shape and dimensional parameters on the crashing behaviors of corrugated sandwich structures and improve the sandwich cores with the trapezoidal and truss-type corrugation configurations for crashworthiness standards. It will also compare the improved crashworthiness of these two different corrugated sandwich structures, thus given some guides to design of sandwich structures. Corrugated metal sandwich cores have verified excellent shock resistant properties, generally due to their high longitudinal stretching and shear strength [26].

\subsubsection{Triangular}

For triangular shaped corrugated-core, several authors already discussed the strength and properties. For example, Buannic et al, [28] studies the homogenization of corrugated sandwich panels. In this studies several shapes of corrugated-cores were selected including triangular shaped. Rejab et al, [14]conducted series of experimental investigations and numerical analyses is presented into the compression response, and subsequent failure modes in corrugated-core sandwich panels based on an aluminum alloy, a glass fiber (GFRP) and a carbon fibre reinforced plastic (CFRP). Tian et al. [29] experimented compression panel comprised of different type of corrugated-core including triangular. Figure 3 below shows the shape of triangular corrugated-cores which is triangle shape.

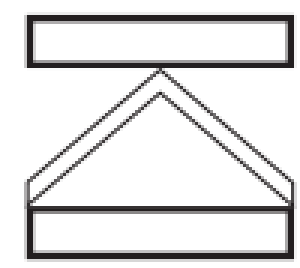

Figure 3: Triangle corrugated-cores (Bartolozzi et al. 2014) [30]

\subsubsection{Sinusoidal}

Bartolozzi et al. [25] investigated sinusoidal corrugatedcores aluminum sandwich structures. The main field of application of these aluminum structures is the transport area, e.g. in the automotive industry, where energy conservation, lightweight manufacture and recycling are critical requirements. Also marine internal applications are common, since these panels provide good structural performance with small thicknesses and can also be simply supplied in semi-finished components. The properties of the equivalent material are determined both analytically and numerically for the chosen ReissnerMindlin orthotropic representation. The two derived models are compared in a comprehensive parametric study to validate the computationally much cheaper analytical formulation. Furthermore, a validation of the equivalent models is done based on the bending stiffness per unit width of the sandwich panel. Finally, the acoustic behavior of the structure is investigated comparing the reduced layered model with the fully detailed 3D model. Figure 4 shows sinusoidal corrugated-cores that have radius in the shape.

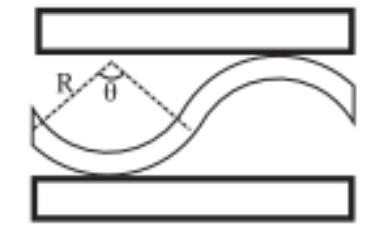

Figure 4: Sinusoidal corrugated-cores (Bartolozzi et al. 2014)[30]

\subsubsection{Trapezoidal}

The finite element modeling (FEM) and the impact responses of stacked trapezoidal corrugated aluminum core and aluminum sheet interlayer sandwich structures was studied by Kiliçaslan et al. [31]. Thill et al. [32] investigated about trapezoidal corrugated aramid/epoxy laminates under huge tensile displacements transverse to the corrugation direction. Figure 5 shows trapezoidal corrugated-cores that have trapezium in shape with the angle. Kiliçaslan et al. [33] do numerical studies and experimental on the quasi-static and dynamic crushing responses of multilayer trapezoidal aluminum corrugated sandwiches.

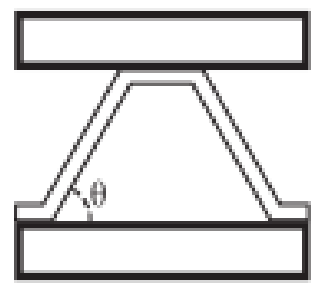

Figure 5: Trapezoidal corrugated-cores (Bartolozzi et al. 2014) [30]

\section{Conclusions}

Sandwich structures are commonly based on polymeric foam and honeycomb. Sandwich structure based on corrugated-core with different shape of corrugated-core, which is trapezoidal, triangle and sinusoidal was discussed in this paper. The important features of this corrugated-core sandwich structures is its high strength to weight ratio. Recent researchers already make several experiments to investigate the strength of the corrugatedcore sandwich structures. Less of the research focus on trapezoidal sandwich structure which offer high strength in compression test and shear test. Furthermore, due to their high flexural stiffness to weight ratio, the structure usually used in making aircraft, marine ship, bridge and other application. From previous study, it showed that corrugated-core offer shear strengths when tested in the longitudinal direction which can be compare with square honeycombs and significantly greater than diamond cores and more traditional foam cores.

\section{Acknowledgements}

The authors would like to be obliged to University Malaysia Pahang for providing laboratory facilities and financial assistance under project no. RDU140116. 


\section{References}

[1] D. G. Luchinsky, V. Hafiychuk, V. Smelyanskiy, R. Tyson, J. L. Walker, and J. L. Miller, "High-fidelity modeling for health monitoring in honeycomb sandwich structures," in Aerospace Conference, 2011 IEEE, (2011), pp. 1-7.

[2] Y. A. Bruno Castanié, Christophe Bouvet, JeanJacques Barrau, "Core crush criterion to determine the strength of sandwich composite structures subjected to compression after impact 2008 Composite Structures.pdf," Composite Structures, pp. 243-250, (2008).

[3] H. N. Wadley, "Multifunctional periodic cellular metals," Philos Trans A Math Phys Eng Sci, vol. 364, pp. 31-68, Jan 15 (2006).

[4] A. Mamalis, D. Manolakos, M. Ioannidis, P. Kostazos, and D. Papapostolou, "Axial collapse of hybrid square sandwich composite tubular components with corrugated core: numerical modelling," Composite structures, vol. 58, pp. 571-582, (2002).

[5] S. Belouettar, A. Abbadi, Z. Azari, R. Belouettar, and P. Freres, "Experimental investigation of static and fatigue behaviour of composites honeycomb materials using four point bending tests," Composite Structures, vol. 87, pp. 265-273, (2009).

[6] H. P. Konka, M. A. Wahab, and K. Lian, "On Mechanical Properties of Composite Sandwich Structures With Embedded Piezoelectric Fiber Composite Sensors," Journal of Engineering Materials and Technology, vol. 134, p. 011010 , (2012).

[7] L. Librescu and T. Hause, "Recent developments in the modeling and behavior of advanced sandwich constructions: a survey," Composite structures, vol. 48, pp. 1-17, (2000).

[8] S. Heimbs, "Virtual testing of sandwich core structures using dynamic finite element simulations," Computational Materials Science, vol. 45, pp. 205-216, (2009).

[9] F. Abbassi, S. Mistou, and A. Zghal, "Failure analysis based on microvoid growth for sheet metal during uniaxial and biaxial tensile tests," Materials \& Design, vol. 49, pp. 638-646, (2013).

[10] H. A. Katzman, R. M. Castaneda, and H. S. Lee, "Moisture diffusion in composite sandwich structures," Composites Part A: Applied Science and Manufacturing, vol. 39, pp. 887-892, (2008).

[11] C. C. Foo, G. B. Chai, and L. K. Seah, "Mechanical properties of Nomex material and Nomex honeycomb structure," Composite Structures, vol. 80, pp. 588-594, (2007).

[12] A. Petras and M. Sutcliffe, "Failure mode maps for honeycomb sandwich panels," Composite Structures, vol. 44, pp. 237-252, (1999).
H. Zhao, I. Elnasri, and Y. Girard, "Perforation of aluminium foam core sandwich panels under impact loading-An experimental study," International Journal of Impact Engineering, vol. 34, pp. 1246-1257, (2007).

[14] M. R. M. Rejab and W. J. Cantwell, "The mechanical behaviour of corrugated-core sandwich panels," Composites Part B: Engineering, vol. 47, pp. 267-277, (2013).

[15] M. Yamashita and M. Gotoh, "Impact behavior of honeycomb structures with various cell specifications-numerical simulation and experiment," International Journal of Impact Engineering, vol. 32, pp. 618-630, (2005).

[16] L. Aktay, A. F. Johnson, and B.-H. Kröplin, "Numerical modelling of honeycomb core crush behaviour," Engineering Fracture Mechanics, vol. 75, pp. 2616-2630, (2008).

[17] A. Abbadi, Y. Koutsawa, A. Carmasol, S. Belouettar, and Z. Azari, "Experimental and numerical characterization of honeycomb sandwich composite panels," Simulation Modelling Practice and Theory, vol. 17, pp. 1533-1547, (2009).

[18] V. N. Burlayenko and T. Sadowski, "Analysis of structural performance of sandwich plates with foam-filled aluminum hexagonal honeycomb core," Computational Materials Science, vol. 45, pp. 658-662, (2009).

[19] M. Burman and D. Zenkert, "Fatigue of foam core sandwich beams-1: undamaged specimens," International journal of fatigue, vol. 19, pp. 551-561, (1997).

[20] C. Chen, A. Harte, and N. Fleck, "The plastic collapse of sandwich beams with a metallic foam core," International Journal of Mechanical Sciences, vol. 43, pp. 1483-1506, (2001).

[21] V. Crupi and R. Montanini, "Aluminium foam sandwiches collapse modes under static and dynamic three-point bending," International Journal of Impact Engineering, vol. 34, pp. 509521, (2007).

[22] A.-M. Harte, N. Fleck, and M. Ashby, "The fatigue strength of sandwich beams with an aluminium alloy foam core," International Journal of Fatigue, vol. 23, pp. 499-507, (2001).

[23] S. Kazemahvazi, R. Ben, D. Vikram, and D. Zenkert, "Impact properties of corrugated composite sandwich cores," in International Conference on Sandwich Structures, ICSS9, (2010).

[24] W.-S. Chang, E. Ventsel, T. Krauthammer, and J. John, "Bending behavior of corrugated-core sandwich plates," Composite Structures, vol. 70, pp. 81-89, (2005).

[25] G. Bartolozzi, M. Pierini, U. Orrenius, and N. Baldanzini, "An equivalent material formulation for sinusoidal corrugated cores of structural sandwich panels," Composite Structures, vol. 100, pp. 173-185, (2013).

[26] F. Côté, V. S. Deshpande, N. A. Fleck, and A. G. Evans, "The compressive and shear responses 
of corrugated and diamond lattice materials," International Journal of Solids and Structures, vol. 43, pp. 6220-6242, (2006).

[27] S. Hou, S. Zhao, L. Ren, X. Han, and Q. Li, "Crashworthiness optimization of corrugated sandwich panels," Materials \& Design, vol. 51, pp. 1071-1084, (2013).

[28] N. Buannic, P. Cartraud, and T. Quesnel, "Homogenization of corrugated core sandwich panels," Composite Structures, vol. 59, pp. 299312, (2003).

[29] Y. S. Tian and T. J. Lu, "Optimal design of compression corrugated panels," Thin-Walled Structures, vol. 43, pp. 477-498, (2005).

[30] G. Bartolozzi, N. Baldanzini, and M. Pierini, "Equivalent properties for corrugated cores of sandwich structures: A general analytical method," Composite Structures, vol. 108, pp. 736-746, (2014).

[31] C. Kılıçaslan, M. Güden, İ. K. Odacı, and A. Taşdemirci, "The impact responses and the finite element modeling of layered trapezoidal corrugated aluminum core and aluminum sheet interlayer sandwich structures," Materials \& Design, vol. 46, pp. 121-133, (2013).

[32] C. Thill, J. A. Etches, I. P. Bond, K. D. Potter, P. M. Weaver, and M. R. Wisnom, "Investigation of trapezoidal corrugated aramid/epoxy laminates under large tensile displacements transverse to the corrugation direction," Composites Part A: Applied Science and Manufacturing, vol. 41, pp. 168-176, (2010).

[33] C. Kilıçaslan, M. Güden, İ. K. Odac1, and A. Taşdemirci, "Experimental and numerical studies on the quasi-static and dynamic crushing responses of multi-layer trapezoidal aluminum corrugated sandwiches," Thin-Walled Structures, vol. 78, pp. 70-78, (2014). 